1 Secretaria Municipal de Saúde do Rio de Janeiro (SMS-RJ) - Rio de Janeiro, Brasil.

pharmacistnath@gmail.com

2 Fundação Oswaldo Cruz (Fiocruz), Escola Naciona de Saúde Pública Sérgio Arouca (Ensp), Núcleo de Assistência Farmacêutica (NAF) - Rio de Janeiro,

Brasil.

vera@ensp.fiocruz.br

${ }^{3}$ Fundação Oswaldo Cruz (Fiocruz), Escola Nacional de Saúde Pública Sérgio Arouca (Ensp), Departamento de Endemias Samuel Pessoa Rio de Janeiro, Brasil. marly@ensp.fiocruz.br

\section{Serviços farmacêuticos na atenção primária no município do Rio de Janeiro: um estudo de avaliabilidade}

\author{
Pharmaceutical services at primary care in the municipality of Rio de \\ Janeiro: an evaluability assessment
}

Nathália Cano Pereira', Vera Lucia Luiza², Marly Marques da Cruz ${ }^{\mathbf{3}}$

RESUMO Objetivou-se descrever o desenvolvimento de etapas do estudo de avaliabilidade dos serviços farmacêuticos na Atenção Primária à Saúde do município do Rio de Janeiro. Pesquisa documental, com entrevistas realizadas para identificar objetivos, metas e envolvidos nos serviços na atenção primária. Estes subsidiaram a construção do Modelo Lógico de intervenção que priorizou o desenvolvimento de atividades voltadas para o cuidado com o usuário e as comunidades, considerando duas células operacionais: gestão técnica do medicamento e gestão do cuidado. $\mathrm{O}$ estudo de avaliabilidade mostrou-se fundamental por possibilitar organizar a construção do modelo do serviço farmacêutico com base em seus pressupostos.

PALAVRAS-CHAVE Serviços comunitários de farmácia; Atenção Primária à Saúde; Avaliação em saúde.

ABSTRACT It was aimed to describe the stages development of the evaluability assessment of Pharmaceutical Services in Primary Health Care in the municipality of Rio de Janeiro. Documentary research, with interviews performed to identify the objectives, goals and stakeholders of the Pharmaceutical Service. The latter subsidized the construction of the Logical Model of the intervention. The Logical Model has prioritized the development of activities aimed at care over individual users and communities, by considering two operating cells: technical management of medicine and management care. The evaluability assessment was fundamental for enabling to organize the construction of the Pharmaceutical Services of Logical Model based on their assumptions.

KEYWORDS Community pharmacy services; Primary Health Care; Health evaluation. 


\section{Introdução}

Grandes esforços têm sido desenvolvidos, em âmbito mundial, para garantir acesso aos medicamentos essenciais e ampliar a difusão de políticas nacionais de medicamentos. Apesar disso, uma grande proporção da população ainda não tem essa necessidade garantida. Paradoxalmente, o consumo e a produção de medicamentos crescem a cada ano, exibindo as iniquidades no acesso, o desperdício e o uso inadequado. Esses problemas não estão restritos apenas à população em geral, mas também envolvem prescritores e outros profissionais de saúde, o que gera consequências sanitárias, econômicas e sociais para serviços e sistemas de saúde (OPAS, 2013).

Essas são algumas das razões pelas quais a Organização Pan-Americana da Saúde (Opas) propõe a reorientação de políticas e estratégias relacionadas a medicamentos, que, até o presente momento, estiveram centradas no produto (OPAS, 2009). A nova visão propõe como foco os indivíduos, a família e a comunidade, com suas necessidades e condições de saúde, tendo o medicamento e os Serviços Farmacêuticos (Sefar) entre os elementos fundamentais para a garantia da atenção integral e contínua (RICIERI ET AL., 2006).
Os Sefar na Atenção Primária à Saúde (APS) são definidos como um conjunto de ações no sistema de saúde que busca garantir atenção integral, integrada e contínua às necessidades e aos problemas de saúde da população, tanto no âmbito individual quanto no coletivo, tendo o medicamento como um dos elementos essenciais, contribuindo para seu acesso equitativo e uso racional. Essas ações devem ser desenvolvidas por farmacêutico ou sob sua coordenação, incorporado à equipe de saúde com vistas à melhoria da qualidade de vida da população. A reflexão sobre essa nova proposta enseja estabelecer sua relação com os valores e princípios da APS e reconhecer que os Sefar têm sua máxima expressão no nível da microgestão (OPAS, 2013).

Ainda que o termo Serviços Farmacêuticos tenha historicamente se originado da tradução de pharmaceutical services, do inglês, ou de servicios farmacêuticos, do espanhol, a expressão Assistência Farmacêutica (AF) ganhou maior abrangência no Brasil, e seu conceito incluiu aspectos como pesquisa, desenvolvimento de produtos, produção de fármacos etc. Dessa maneira, no Brasil, conceitos distintos de AF e Sefar têm sido adotados, entendendo Sefar como um conjunto de ações contidas na AF, que envolvem atividades e processos mais relacionados às unidades e aos serviços de saúde (figura 1).

Figura 1. Organização dos Serviços Farmacêuticos na Atenção Primária à Saúde

Indivíduo, família e comunidade.

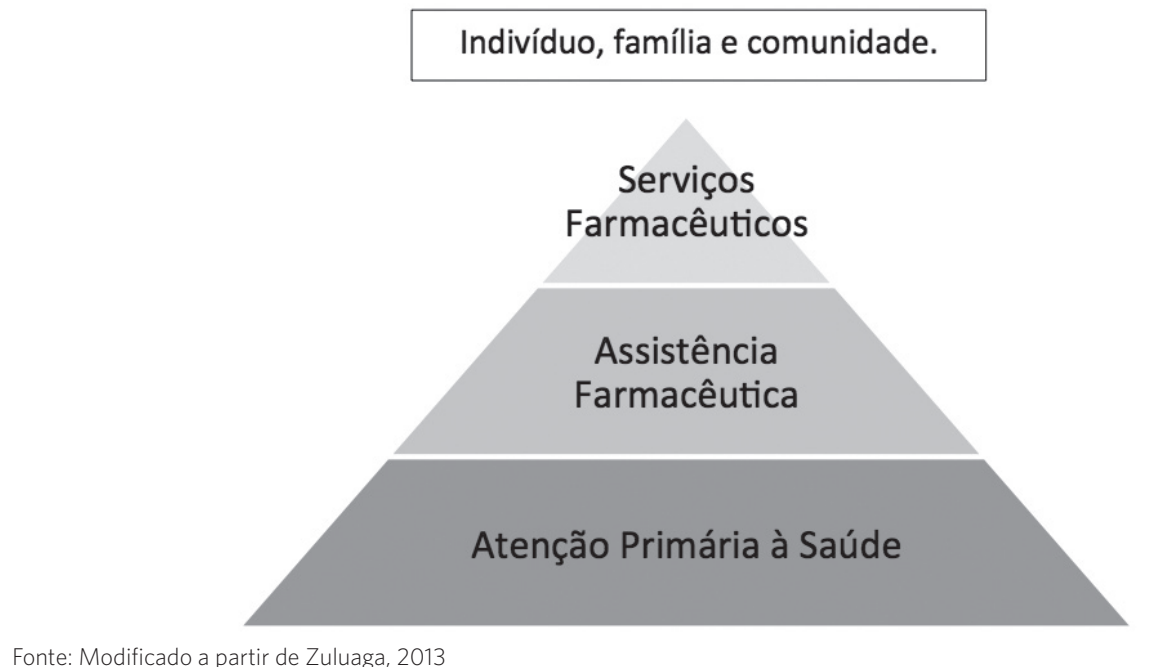

Fonte: Modificado a partir de Zuluaga, 2013 
A organização e a estruturação da AF têm priorizado a execução de ações no nível da macrogestão municipal, desenvolvendo atividades restritas aos componentes técnicos ligados ao ciclo logístico do medicamento: seleção, programação, aquisição, armazenamento, distribuição e dispensação (ARAUjo; UETA; FREITAS, 2005; MARIN ET AL., 2003). Apesar dos grandes marcos referenciais do modelo de AF vigente, as atuais políticas têm se mostrado ineficientes na obtenção de resultados satisfatórios com relação ao uso racional de medicamentos, com ações ainda desarticuladas do processo de cuidado em saúde (CORRER; OTUKI; SOLER, 2011).

As ações da AF restritas à provisão do acesso aos medicamentos não dão conta de responder à necessidade da atenção integral à saúde, sobretudo na APS, onde a necessidade de abordagem do indivíduo em sua singularidade sociocultural é imperiosa (ARAUJO; UETA; FREITAS, 2005; BRASIL, 2011A). Assim, faz-se necessário concentrar esforços para a implantação de Sefar integrados aos serviços de saúde, tendo como objetivos: garantir a disponibilidade adequada de medicamentos, sua qualidade e conservação; prestar serviços assistenciais, com foco na efetividade e na segurança da terapêutica; avaliar, obter e difundir informações sobre medicamentos e sobre saúde na perspectiva da educação em saúde e da educação permanente das equipes de saúde (OPAS, 2013; BRASIL, 2009A).

Atualmente, o Departamento de AF do Ministério da Saúde tem repensado o papel do farmacêutico na APS brasileira, reconhecendo sua centralidade na viabilização do modelo de Sefar proposto. A presença do farmacêutico na Estratégia Saúde da Família (ESF), ora vinculada à estrutura de Núcleo de Apoio à Saúde da Família (Nasf), ora em alguma outra conformação, faz parte de uma nova etapa de construção e consolidação dos Sefar no Brasil. É o momento de repensar conhecimentos e propor novas atribuições e atividades mais relacionadas com o trabalho em equipe e o cuidado ao usuário (ARAUJO;
UETA; FREITAS, 2005). Sendo assim, é fundamental investir na construção de ferramentas de gestão que possam contribuir para a operacionalização desse novo modelo de Sefar, facilitando a comunicação de seus objetivos e práticas aos gestores, profissionais e usuários.

Em que pesem os esforços realizados nas diferentes esferas de gestão, ainda que sejam escassas as evidências geradas no País (MENDES ET AL., 2014), há um razoável consenso entre os atores com experiência nacional (BRASIL, 2009A; VIEIRA, 2007) acerca da fragilidade dos modelos de Sefar em operação na maioria dos municípios. Mendes et al. (2014), em estudo realizado com dados de 38.812 unidades básicas brasileiras, afirmam que, embora $75,3 \%$ das unidades dispensem medicamentos, a disponibilidade média desses produtos ficou abaixo do valor aceitável, de $80 \%$, proposto pela OMS. Além disso, das 11.144 unidades básicas que dispensavam medicamentos e aderiram ao Programa Nacional de Melhoria do Acesso e da Qualidade da Atenção Básica (PMAQ-AB), apenas 20,1\% contavam com apoio matricial de farmacêutico.

O município do Rio de Janeiro, como parte dos esforços de renovação gerencial na atenção primária e em convergência com a proposta da Opas e do MS, tem desenvolvido estratégias para consolidar a implantação dos Sefar diretamente vinculados às unidades de saúde e com presença de profissional farmacêutico. Por se tratar de um processo de implantação em curso, requer planejamento e monitoramento contínuos com ferramentas de gestão capazes de subsidiar a tomada de decisões gerenciais em tempo oportuno (CRUZ, 2011), apontando evidências para redirecionamento e melhoria do Sefar na APS.

A avaliação e o monitoramento compõem, juntamente com o planejamento e a implementação, os principais componentes do processo gerencial (CRUZ, 2011). O Estudo de Avaliabilidade (EA) é formado por um conjunto de procedimentos que devem 
anteceder a avaliação, fortalecendo seu uso para a tomada de decisão. É, portanto, etapa fundamental no processo avaliativo (NATAL ET AL., 2010). Esse tipo de estudo é precípuo quando se busca o aprimoramento das intervenções, pois estas preveem o esclarecimento dos propósitos da avaliação, assim como a participação dos gestores e demais interessados nas diferentes etapas do processo (BEZERRA; CAZARIN; ALVES, 2010). Considerase o EA como uma boa ferramenta de gestão por favorecer a construção de entendimentos entre os envolvidos sobre a natureza e os objetivos da intervenção, aumentado às possibilidades dos resultados da avaliação (THURSTON; RAMALIU, 2005).

Este artigo pretende apresentar o desenvolvimento de etapas do EA dos Sefar na APS, com vistas a contribuir com uma ferramenta de gestão útil para os municípios e para a definição das atribuições e atividades dos farmacêuticos vinculados aos Sefar.

\section{Método}

Para desenvolvimento do EA foram consideradas algumas etapas da abordagem proposta por Trurston e Ramaliu (2005), sendo elas: identificar metas e objetivos da intervenção; definir os potenciais envolvidos; identificar e analisar documentos e delinear a intervenção - Modelo Lógico (ML). Para a construção das etapas, foram utilizadas como ferramentas metodológicas a pesquisa documental e a entrevista semiestruturada com os atores-chave identificados. A pesquisa documental e as entrevistas convergiram na importância da implantação simultânea de um conjunto mínimo de ações gerenciais e clínicas para que a intervenção alcance seus objetivos primários de acesso e promoção do uso racional.

O município do Rio de Janeiro foi escolhido como local do estudo devido ao interesse na construção de um modelo de desempenho da atuação dos Sefar na APS desse município.
Esse levantamento foi realizado no período de maio a julho de 2013.

O primeiro passo para a construção do EA foi identificar os objetivos e as metas dos Sefar nos documentos e nas entrevistas. Foram selecionados documentos que tratavam da $\mathrm{AF}$ na APS em diversos contextos - internacional, nacional e municipal. Os objetivos gerais, específicos e as metas identificadas nos documentos foram analisados e sistematizados. Para a entrevista, foi utilizado um roteiro semiestruturado aplicado a atores-chave envolvidos no contexto da $\mathrm{AF}$ municipal, que foram indagados sobre os objetivos e as metas da intervenção, bem como acerca dos resultados esperados com a sua implementação.

A escolha dos sujeitos para a entrevista levou em consideração seu envolvimento na intervenção, considerando seu potencial e sua capacidade de interferir no processo de implementação e na tomada de decisão para a melhoria da AF na APS do município estudado, com representação das três esferas: federal, estadual e municipal. De posse das falas dos atores, as ideias centrais foram categorizadas pela análise de conteúdo proposta por Bardin (2011).

As mesmas fontes de dados permitiram a execução do segundo passo, que consistiu na identificação dos potenciais envolvidos e interessados na implantação da intervenção. Deu-se foco àqueles com envolvimento relevante no $\mathrm{ML}$, o que implica aqueles responsáveis pela provisão dos insumos, pela execução das atividades e os sujeitos que recebem os resultados.

O terceiro passo consistiu no delineamento da intervenção, ensejando a organização do conjunto de elementos no ML propriamente dito, ferramenta bastante útil que permite, de maneira visual e sistemática, apresentar as relações entre recursos necessários, atividades e efeitos (produtos, resultados e impacto) que se pretende alcançar com a intervenção (BEZERRA; CAZARIN; ALVES, 2010). Facilitar a comunicação do propósito fundamental da intervenção e apresentar a sua teoria de implantação 
são vantagens precípuas do $\mathrm{ML}$, considerado um dos principais produtos do Estudo de Avaliabilidade, pois permite descrever de forma clara e coerente o funcionamento da intervenção (NATAL ET AL., 2010).

O projeto foi aprovado pelos Comitês de Ética em Pesquisa da Escola Nacional de Saúde Pública, CAAE: 12443313.7.0000.5240, e da Secretaria Municipal de Saúde do Rio de Janeiro, Protocolo de Pesquisa $n^{0}$ 56/2013. Todos os entrevistados foram solicitados a manifestar sua anuência por meio da assinatura de Termo de Consentimento Livre e Esclarecido, sendo informados da possibilidade de sua identificação, tendo em vista a natureza pública de seus cargos.

\section{Resultados}

A pesquisa documental considerou documento de posição de organismo internacional (OPAS, 2013), diretrizes ministeriais (BRASIL, 2006, 2009A, 2009B), diretrizes municipais (RIO DE JANEIRO, 2011) específicas quanto aos Sefar e o Plano Municipal de Saúde (RIO DE JANEIRO, 2009).

No total, foram entrevistados seis atores-chave: o Assessor Técnico da Assistência
Farmacêutica do Conselho de Secretários Municipais em Saúde do Rio de Janeiro (Cosems-RJ) e apoiador do Departamento de Assistência Farmacêutica/Ministério da Saúde; o Superintendente da Assistência Farmacêutica da Secretaria Estadual de Saúde; o Subsecretário de atenção primária, Promoção e Vigilância em Saúde; o Coordenador da Assistência Farmacêutica Municipal em exercício e seu antecessor; e um Coordenador Regional da Assistência Farmacêutica no município. As entrevistas duraram, em média, 40 minutos.

\section{Identificação dos objetivos e metas dos Serviços Farmacêuticos}

De maneira consensual, os documentos analisados, independentemente de sua abrangência quanto à micro ou macrogestão, apresentaram como objetivo geral dos Sefar na APS: 'promover acesso a medicamentos essenciais e seu uso racional'. Além disso, foram encontrados em três (BRASIL, 2006; MARIN ET AL., 2003; RIO, 2011) dos sete documentos analisados objetivos específicos relacionados com os componentes técnicos do ciclo da AF (quadro 1).

Quadro 1. Apresenta a síntese dos objetivos específicos organizados por componente técnico dos Serviços Farmacêuticos encontrados na pesquisa documental

\begin{tabular}{|c|c|c|}
\hline Componente Técnico & Documento & Objetivos dos Serviços Farmacêuticos \\
\hline \multirow[t]{2}{*}{ Seleção } & $A^{\star}$ & $\begin{array}{l}\text { - Uniformizar condutas terapêuticas. } \\
\text { - Melhorar o acesso aos medicamentos selecionados. } \\
\text { - Contribuir para promoção do uso racional de medicamentos. } \\
\text { - Facilitar a integração multidisciplinar, envolvendo os profissionais de saúde na tomada de decisões. } \\
\text { - Favorecer o processo de educação continuada e atualização dos profissionais, além do uso apropriado dos } \\
\text { medicamentos. }\end{array}$ \\
\hline & $B^{\star}$ & - Promover do uso racional de medicamentos. \\
\hline Programação & A & $\begin{array}{l}\text { - Identificar quantidades necessárias de medicamentos para o atendimento às demandas da população. } \\
\text { - Evitar aquisições desnecessárias, perdas e descontinuidade do abastecimento. } \\
\text { - Definir prioridades e quantidades a serem adquiridas diante da disponibilidade de recurso. }\end{array}$ \\
\hline Aquisição & A & $\begin{array}{l}\text { - Suprir necessidades de medicamentos em quantidade, qualidade e menor custo-efetividade. } \\
\text { - Manter a regularidade do sistema de abastecimento. }\end{array}$ \\
\hline
\end{tabular}




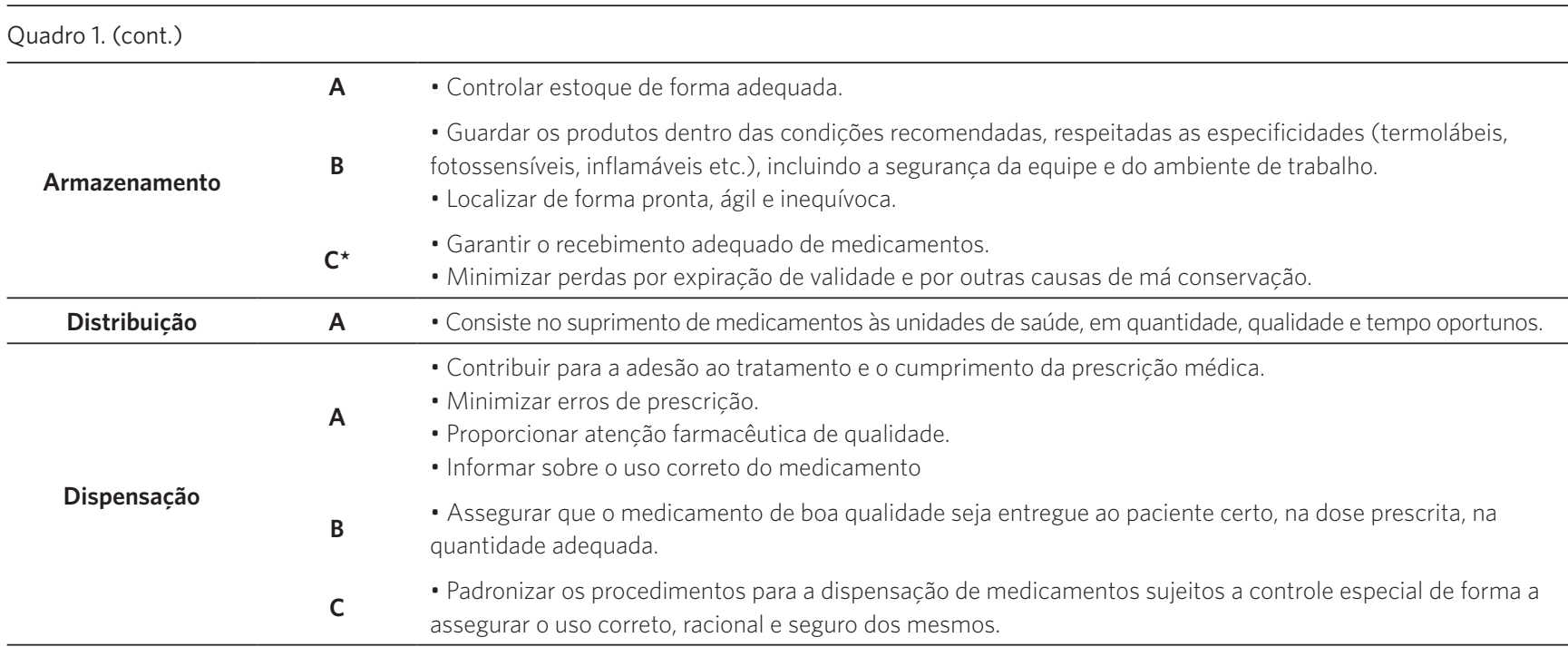

Fonte: Elaboração própria

$A^{\star}$ : Assistência Farmacêutica na Atenção Básica: Instruções Técnicas para sua organização (BRASIL, 2006)

$B^{*}$ : Assistência Farmacêutica para gerentes municipais (Marin et al., 2003)

$C^{\star}$ : Manual de Instruções, Diretrizes e Procedimentos Operacionais da Assistência Farmacêutica (RIO DE JANEIRO, 2011)

As metas para os Sefar foram explicitadas apenas no documento (RIO DE JANEIRO, 2009) elaborado pela Secretaria Municipal de Saúde (SMS). Foram identificadas nove metas relacionadas à $\mathrm{AF}$, sendo três ligadas ao Sefar: 1) atender a $100 \%$ da demanda de medicamentos padronizados para a Atenção Básica; 2) produzir e distribuir material educativo sobre o uso de medicamentos a $100 \%$ dos pacientes que tiverem acesso a medicamentos; 3) implantar seguimento farmacoterapêutico em todas as Unidades Básicas de Saúde (UBS).
Nas entrevistas, as ideias centrais das falas referentes aos objetivos foram organizadas como 'ligadas aos objetivos gerais e aos objetivos específicos da intervenção'. Já as metas e os resultados esperados foram categorizados em dois grandes grupos: 'gestão técnica do medicamento', com as ideias ligadas às ações de logística e provisão do acesso; e 'gestão do cuidado ao usuário', com as ideias referentes à relação farmacêutico-usuário-equipes de saúde. Em cada um dos grupos, as ideias foram classificadas como sendo de curto, médio ou longo prazo (quadro 2). 
Quadro 2. Ideias centrais presentes nas falas dos entrevistados acerca dos objetivos, metas e resultados esperados dos Serviços Farmacêuticos na atenção primária no município do Rio de Janeiro, 2013

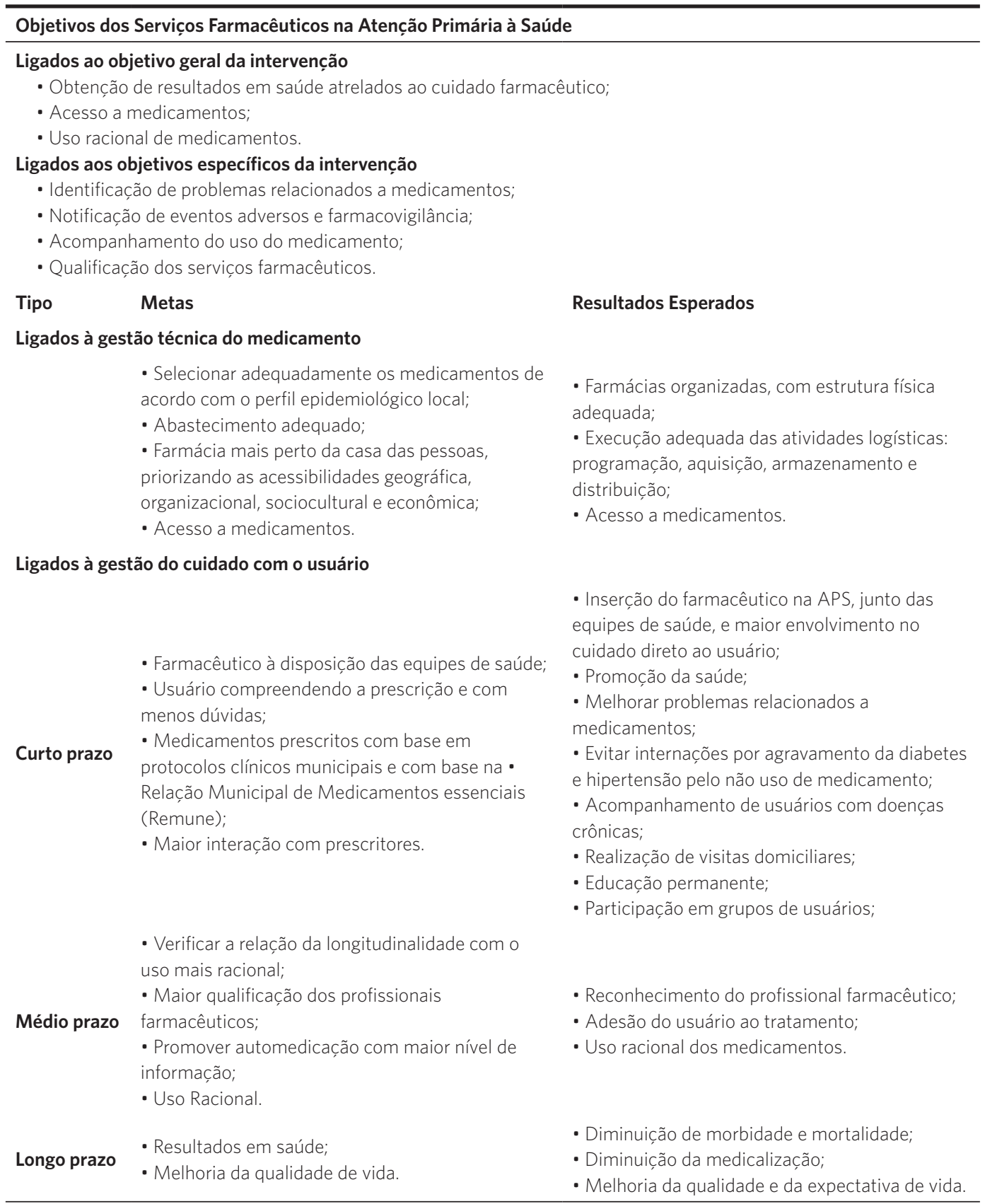

\section{Principais envolvidos e interessados na AF municipal}

Os principais envolvidos e interessados identificados foram organizados em 3 grupos, tendo em conta o envolvimento dos atores nos Sefar.
São eles: a) os responsáveis pela provisão de infraestrutura e insumos: Subsecretário de atenção primária, Promoção e Vigilância em Saúde; o Coordenador da Assistência Farmacêutica Municipal; os Coordenadores de Área de Planejamento; e Coordenadores 
Regionais da Assistência Farmacêutica; b) os responsáveis pela execução das atividades: gerentes; farmacêuticos; e auxiliares de farmácia das unidades da APS; e c) os sujeitos que sofrem a ação dos Sefar e recebem seus resultados: usuários do território.
A fim de esclarecer o papel de cada um dos principais envolvidos na intervenção, seus interesses na implantação e qual o uso provável do ML, as informações foram sintetizadas e organizadas conforme a atuação nos Sefar (quadro 3).

Quadro 3. Principais envolvidos na implantação dos Serviços Farmacêuticos no município do Rio de Janeiro e seus respectivos papéis na intervenção, interesse na implantação e seu uso provável do Modelo Lógico. Rio de Janeiro, 2013

\begin{tabular}{|c|c|c|c|}
\hline Atores & Papel na intervenção & Interesse na implantação & Uso provável do Modelo Lógico \\
\hline \multicolumn{4}{|c|}{ Provisão de infraestrutura e insumos } \\
\hline $\begin{array}{l}\text { Subsecretário de } \\
\text { Atenção Primária, } \\
\text { Promoção e } \\
\text { Vigilância em Saúde }\end{array}$ & $\begin{array}{l}\text { - Financiar, definir estratégias de } \\
\text { funcionamento e acompanhar a } \\
\text { execução das ações de AF na APS no } \\
\text { município do Rio de Janeiro. }\end{array}$ & $\begin{array}{l}\text { - Garantir aos munícipes o direito } \\
\text { ao tratamento integral, gratuito e } \\
\text { de qualidade, provendo acesso a } \\
\text { medicamentos e seu uso racional. }\end{array}$ & $\begin{array}{l}\text { - Acompanhar a aplicação dos recursos e } \\
\text { seus resultados; } \\
\text { - Melhoria do processo de gestão. }\end{array}$ \\
\hline $\begin{array}{l}\text { Coordenador } \\
\text { da Assistência } \\
\text { Farmacêutica } \\
\text { Municipal }\end{array}$ & $\begin{array}{l}\text { - Definir padrões e normas, organizar, } \\
\text { executar processos de compra de } \\
\text { medicamentos e acompanhar as } \\
\text { ações relacionadas à intervenção no } \\
\text { nível municipal; } \\
\text { - Ensejar a discussão sobre a } \\
\text { importância da AF no nível municipal. }\end{array}$ & $\begin{array}{l}\text { - Garantir consolidação da AF no } \\
\text { município de forma permanente e eficaz; } \\
\text { - Prover acesso a medicamentos } \\
\text { de qualidade, com a presença do } \\
\text { profissional farmacêutico promovendo } \\
\text { seu uso racional. }\end{array}$ & $\begin{array}{l}\text { - Esclarecer os objetivos, metas e } \\
\text { resultados da intervenção; } \\
\text { - Melhorar o processo de gestão; } \\
\text { - Identificar facilitadores e obstáculos do } \\
\text { processo de implantação; } \\
\text { - Acompanhar a aplicação dos recursos e } \\
\text { seus resultados. }\end{array}$ \\
\hline $\begin{array}{l}\text { Coordenador de } \\
\text { Área Programática } \\
\text { Municipal }\end{array}$ & $\begin{array}{l}\text { - Definir local de implantação } \\
\text { dos Sefar, estruturação física e } \\
\text { contratação de recursos humanos; } \\
\text { - Acompanhar a execução das ações } \\
\text { dos Sefar no nível regional. }\end{array}$ & $\begin{array}{l}\text { - Garantir aos munícipes o direito } \\
\text { ao tratamento integral, gratuito e } \\
\text { de qualidade, provendo acesso a } \\
\text { medicamentos e uso racional em uma } \\
\text { área de planejamento; } \\
\text { - Aumentar a satisfação da comunidade } \\
\text { com o serviço prestado. }\end{array}$ & $\begin{array}{l}\text { - Acompanhar a aplicação dos recursos e } \\
\text { seus resultados; } \\
\text { - Melhoria do processo de gestão. }\end{array}$ \\
\hline $\begin{array}{l}\text { Coordenador } \\
\text { Regional da } \\
\text { Assistência } \\
\text { Farmacêutica }\end{array}$ & $\begin{array}{l}\text { - Organizar, executar e acompanhar } \\
\text { as ações relacionadas à intervenção } \\
\text { em nível regional; } \\
\text { - Ensejar a discussão sobre a } \\
\text { importância da AF no nível regional. }\end{array}$ & $\begin{array}{l}\text { - Consolidar a AF em uma área de } \\
\text { planejamento de forma permanente e } \\
\text { eficaz; } \\
\text { - Prover acesso a medicamentos } \\
\text { de qualidade, com a presença do } \\
\text { profissional farmacêutico promovendo } \\
\text { seu uso racional. }\end{array}$ & $\begin{array}{l}\text { - Esclarecer os objetivos, metas e } \\
\text { resultados da intervenção; } \\
\text { Identificar facilitadores e obstáculos do } \\
\text { processo de implantação; } \\
\text { - Acompanhar a aplicação dos recursos e } \\
\text { seus resultados. }\end{array}$ \\
\hline \multicolumn{4}{|c|}{ Execução das atividades } \\
\hline $\begin{array}{l}\text { Gerente do Centro } \\
\text { Municipal de Saúde }\end{array}$ & $\begin{array}{l}\text { - Apoiar as estratégias de } \\
\text { funcionamento dos Sefar, } \\
\text { corresponsabilizar pela boa execução } \\
\text { das atividades previstas pelos } \\
\text { coordenadores, acompanhar e facilitar } \\
\text { a adequada execução do Sefar no } \\
\text { território. }\end{array}$ & $\begin{array}{l}\text { - Garantir aos usuários do território o } \\
\text { direito ao tratamento integral, gratuito } \\
\text { e de qualidade, provendo acesso a } \\
\text { medicamentos e seu uso racional; } \\
\text { - Contar com um profissional } \\
\text { capacitado para resolução de problemas } \\
\text { relacionados com medicamentos; } \\
\text { Prover satisfação da comunidade com o } \\
\text { serviço prestado. }\end{array}$ & $\begin{array}{l}\text { - Conhecimento sobre os Sefar; ser um } \\
\text { instrumento de apoio na tomada de } \\
\text { decisões gerenciais; melhorar a qualidade } \\
\text { do serviço prestado ao usuário e às } \\
\text { equipes e reconhecer resultados positivos } \\
\text { obtidos pela intervenção; } \\
\text { - Divulgar aos usuários e às equipes de } \\
\text { saúde os serviços oferecidos pelos Sefar. }\end{array}$ \\
\hline $\begin{array}{l}\text { Farmacêuticos das } \\
\text { unidades de saúde }\end{array}$ & $\begin{array}{l}\text { - Organizar e executar as ações } \\
\text { da Assistência Farmacêutica nas } \\
\text { unidades de APS. }\end{array}$ & $\begin{array}{l}\text { - Garantir acesso a medicamentos na hora } \\
\text { certa, na dose e quantidade adequada e } \\
\text { mais perto da moradia dos usuários; } \\
\text { - Consolidar o farmacêutico como } \\
\text { profissional de saúde com papel bem } \\
\text { definido no cuidado à saúde das famílias, } \\
\text { indivíduos e comunidades. }\end{array}$ & $\begin{array}{l}\text { - Ampliar a compreensão da intervenção; } \\
\text { - Definir de forma clara quais atividades } \\
\text { e atribuições devem ser desenvolvidas } \\
\text { pelos Sefar e quais resultados devem ser } \\
\text { obtidos; } \\
\text { - Ter um instrumento capaz de esclarecer o } \\
\text { papel do farmacêutico na APS. }\end{array}$ \\
\hline
\end{tabular}




\begin{tabular}{|c|c|c|c|}
\hline \multicolumn{4}{|l|}{ Quadro 3. (cont.) } \\
\hline $\begin{array}{l}\text { Auxiliares de } \\
\text { Farmácia }\end{array}$ & $\begin{array}{l}\text { - Executar as ações da Assistência • } \\
\text { - Farmacêutica nas unidades de APS } \\
\text { conforme orientação do farmacêutico. }\end{array}$ & $\begin{array}{l}\text { - Garantir acesso a medicamentos } \\
\text { na hora certa, na dose e quantidade } \\
\text { adequada e mais perto da moradia dos } \\
\text { usuários. }\end{array}$ & $\begin{array}{l}\text { - Ampliar a compreensão da intervenção, } \\
\text { esclarecendo seus objetivos, metas e } \\
\text { resultados; } \\
\text { - Entender melhor as atividades } \\
\text { desenvolvidas pelos Sefar e quais } \\
\text { resultados devem ser obtidos. }\end{array}$ \\
\hline \multicolumn{4}{|c|}{ Sujeitos que sofrem a ação dos Sefar e recebem seus resultados } \\
\hline Usuário da APS & $\begin{array}{l}\text { - Ser corresponsável pelo seu } \\
\text { processo de cuidado; ter acesso } \\
\text { a medicamentos essenciais e } \\
\text { orientações sobre seu uso. }\end{array}$ & $\begin{array}{l}\text { - Ter um serviço de referência sobre } \\
\text { medicamentos no território onde vive; } \\
\text { - Ter acesso a medicamentos com } \\
\text { qualidade e orientação; } \\
\text { - Verificar o cumprimento dos seus } \\
\text { diretos. }\end{array}$ & $\begin{array}{l}\text { - Ter um instrumento capaz de } \\
\text { esclarecer todas as atividades a serem } \\
\text { desenvolvidas pelos Sefar, servindo } \\
\text { como mecanismo de propor melhorias e } \\
\text { ajustes. }\end{array}$ \\
\hline
\end{tabular}

Fonte: Elaboração própria

Os principais atores identificados como responsáveis pela provisão de infraestrutura e insumos são gestores de dois níveis distintos de tomadas de decisão. Pode-se destacar aqueles da macrogestão, atuantes no que chamamos de nível central da SMS (subsecretário e coordenador da AF); e da meso, vinculados às coordenações de área de planejamento (coordenadores gerais e coordenador regional da AF).

\section{Modelo Lógico dos Serviços Farmacêuticos}

Na construção do Modelo Lógico dos Sefar priorizou-se a racionalidade que se aproximava da realidade no município em foco, porém, com novos elementos voltados para o cuidado ao usuário. Isso se deu devido ao fato de os documentos consultados e as entrevistas preconizarem que as ações dos Sefar sejam centradas nos usuários, nas famílias e na comunidade.
O ML foi organizado considerando-se duas células operacionais: gestão técnica do medicamento e gestão do cuidado (figura 2). Neste estudo, optou-se por utilizar a divisão do modelo nessas células operacionais, visando a demonstrar que a intervenção passa por esses dois momentos operativos distintos. A primeira célula operacional, que está voltada para ações diretamente ligadas ao medicamento, foi organizada em cinco componentes técnicos: seleção, programação, solicitação, armazenamento e distribuição. A segunda célula, voltada às ações ligadas ao uso e ao cuidado com usuários, possui nove componentes técnicos: dispensação; educação em saúde, aconselhamento e medidas de prevenção; tomada de decisões clínicas, diagnósticas e/ou terapêuticas; produção da informação e comunicação; coordenação do cuidado; visão continuada e integral dos processos; trabalho em equipe e relações interprofissionais; educação permanente e formação; e orientação ao cidadão. 
Figura 2. Síntese do Modelo Lógico teórico dos Serviços Farmacêuticos na atenção primária

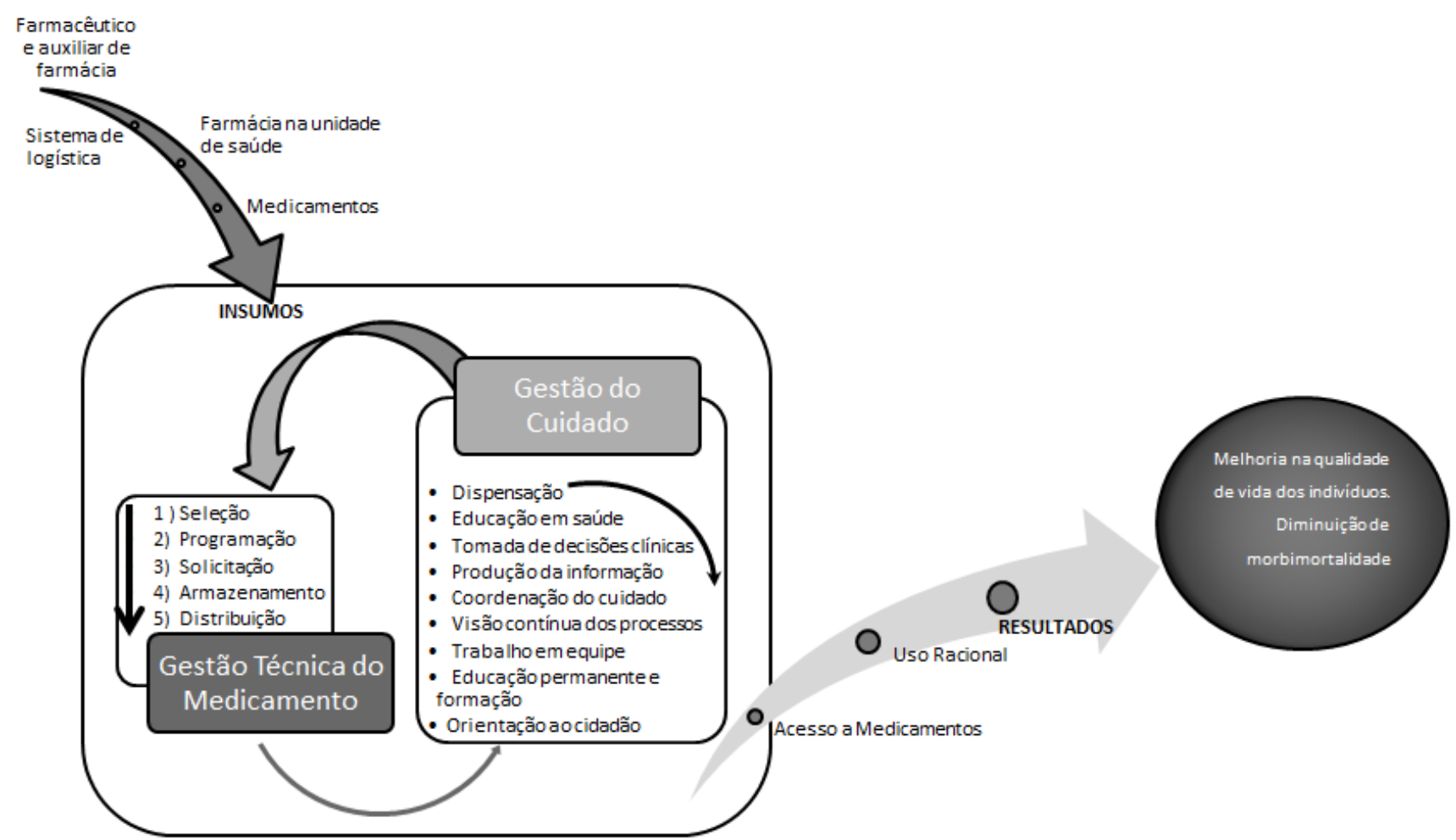

No ML, as células operacionais da 'gestão técnica do medicamento' e da 'gestão do cuidado' estão interligadas e formam um conjunto de componentes técnicos que, juntos, produzem os resultados intermediários esperados da intervenção: acesso a medicamentos e seu uso racional. Cada componente é constituído por vários elementos ou atividades, cujo conjunto propõe uma estratégia para atingir um objetivo específico. Por sua vez, cada elemento se desdobra em um produto, com resultados imediatos e resultados intermediários, que, juntos, produzem o impacto do Sefar. As atividades logísticas, descritas na primeira célula operacional, são consideradas atividades básicas dos Sefar. O desenvolvimento adequado do Sefar depende da realização das atividades vinculadas a cada componente técnico: seleção, programação, solicitação, armazenamento e distribuição. Tais componentes estão interligados numa relação de causa-efeito para a produção dos melhores resultados.

\section{Discussão}

Os objetivos específicos encontrados para o Sefar priorizam atividades mais ligadas às ações gerenciais, ou seja, à gestão técnica do medicamento. Isso se deve ao fato de que tais ações vêm sendo amplamente discutidas nos últimos anos, possibilitando sua definição de forma mais clara nos documentos. Apesar dos documentos abordarem ações voltadas para a relação farmacêutico-usuário, no cotidiano das equipes e das unidades de saúde, definição de objetivos específicos vinculados a essas atividades não foram encontrados. Autores nacionais, como Mendes (2012), ainda consideram a AF como sendo um sistema de apoio às Redes de Atenção à Saúde (RAS) e não algo que faça parte diretamente da gestão da clínica. 
$\mathrm{O}$ fato de não terem sido encontradas metas em outros documentos se deve, principalmente, à abrangência e à função de cada documento dentro do cenário de implantação do Sefar. Os documentos regionais precisam ser mais específicos e práticos do ponto de vista da execução. As metas identificadas foram consideradas incipientes e pouco realistas, pois não dimensionam claramente a adequação entre os recursos disponíveis, o plano de implementação e os efeitos esperados.

A análise das falas e dos textos também permitiu evidenciar que não há uma única forma para implantação dos Sefar, ou seja, não há um modelo definitivo a ser seguido pelos gestores no País. Cada estado, junto com seus municípios, ou até cada município, individualmente, define seu modus operandi. O município do Rio de Janeiro optou por implantar uma proposta de Sefar na qual as farmácias estão vinculadas diretamente à estrutura das unidades de saúde na APS. Além da estrutura física, a inserção do profissional farmacêutico nesses serviços também foi considerada uma prioridade pelos gestores. Essa estruturação favorece a implantação de medidas e ações voltadas à gestão do cuidado aos usuários, indo ao encontro dos objetivos, metas e resultados esperados pelos atores-chave.

Para o sucesso da intervenção, é necessário que tais atores conheçam os objetivos da intervenção e estejam envolvidos com seu processo de implantação. Cada gestor, dentro do seu nível de decisão, desenvolve atividades simultâneas para garantir o adequado funcionamento e a execução dos Sefar, que, por sua vez, ficam sob responsabilidade direta do gerente e do profissional farmacêutico. O gerente tem como papel organizar os meios e os processos de trabalho para que a organização cumpra com seus fins: cuidar da saúde da população, ajudando as pessoas a mudar seus estados de consciência (autonomia) e os trabalhadores a ter mais autonomia na organização de seus processos de trabalho (PESSOA; SANTOS; TORRES, 2011). Esse profissional é responsável por facilitar e garantir, juntamente com o farmacêutico, seu bom funcionamento.

$O$ farmacêutico e sua equipe possuem papel fundamental na implantação dos Sefar na APS. Eles são responsáveis por garantir a efetivação do acesso a medicamento, pois tanto o farmacêutico quanto seus auxiliares promovem ações que possibilitam aos usuários compreender melhor sua saúde, seus medicamentos, seu projeto terapêutico e suas necessidades (OPAS, 2013; RICIERI ET AL., 2006).

Uma forma de instrumentalizar os farmacêuticos e demais profissionais vinculados ao Sefar, a fim de tê-los como promotores de mudanças, é desenvolver instrumentos claros que definam quais são os objetivos, atividades e resultados esperados com a implantação de novos serviços ou programas. $\mathrm{O}$ desenho do ML possibilitou organizar os elementos dos Sefar de uma maneira mais clara acerca da racionalidade da construção da intervenção e das relações causais empregadas na sua elaboração. O detalhamento das atividades em cada componente técnico foi importante para definir de forma clara quais ações devem ser feitas e quais são os insumos necessários para o alcance dos objetivos da intervenção. Segundo Bezerra, Cazarin e Alves (2010), a modelização com nível de detalhamento adequado contribui para o entendimento comum entre os envolvidos na intervenção a respeito do problema que se quer resolver, dos objetivos e dos resultados esperados, favorecendo a identificação de lacunas e resultados fora da realidade ou incoerentes.

O ML, além de ser uma ferramenta útil para os gestores, também pode ser utilizado como uma ferramenta para apresentar aos profissionais de saúde o que foi previsto e será realizado. Entende-se como papel dos gestores nos serviços de saúde democratizar a informação a fim de viabilizar uma participação ativa dos usuários e profissionais no Sistema Único de Saúde (SUS). 
Neste sentido, destaca-se a importância estratégica do conceito de empoderamento (empowerment), definido como o processo através do qual pessoas e comunidades adquirem maior controle sobre as decisões e ações que afetam sua saúde (MARTINS ET AL., 2008).

Embora a divisão em células operacionais seja uma forma estratégica de organizar o $\mathrm{ML}$, cabe ressaltar o caráter inter-relacional dos componentes técnicos e sua aplicação na perspectiva da microgestão, no nível das unidades de saúde de APS.

Na 'seleção', o farmacêutico escolhe quais medicamentos devem ser programados e solicitados para sua unidade. Essa atividade deve levar em conta o perfil epidemiológico do local, bem como a opinião dos prescritores vinculados à unidade de saúde. $\mathrm{Na}$ 'programação', é feita uma estimativa de quanto será consumido mensalmente nas unidades. Dados de consumo, estoque máximo, mínimo, reserva e demanda reprimida precisam ser considerados. De posse das quantidades estimadas, é realizada a 'solicitação' ao Núcleo de Assistência Farmacêutica regional, por meio de planilha eletrônica, dentro do prazo determinado. Ao receber os medicamentos solicitados na data prevista, todas as medidas de conservação de produtos e controle de estoque devem ser seguidas para que seja garantida a qualidade dos medicamentos até sua entrega ao usuário. Após a guarda do medicamento, existem dois mecanismos pelos quais os medicamentos chegam até o usuário: a distribuição e a dispensação.

A 'distribuição' se dá pelo abastecimento de setores da unidade de saúde, como o de observação clínica e a sala de curativos, que necessitam de medicamentos para atendimento de casos de urgência e acompanhamento de usuários com feridas. Esse abastecimento é feito periodicamente e conta com a colaboração de outros profissionais de saúde para a manutenção de estoque mínimo dos setores, garantia de conservação e validade. Nesse caso, embora os usuários tenham acesso aos medicamentos no momento de necessidade, a entrega não é feita diretamente a eles.

A 'gestão do cuidado' possui um número maior de componentes técnicos e de atividades em comparação com a gestão técnica do medicamento. Isso se dá porque esse modelo propõe uma maior dedicação dos Sefar às ações ligadas diretamente ao uso do medicamento e ao usuário. Nessa célula, a dispensação é considerada o principal ato disparador das ações de cuidado, sendo por meio dele que o usuário tem acesso a seus medicamentos e que o farmacêutico inicia sua abordagem assistencial. O bom desempenho da dispensação influencia de forma direta a saúde dos usuários, principalmente por ser o último momento em que há contato do usuário com um profissional de saúde, antes do início do tratamento (BRASIL, 2009A; MARIN ET AL., 2003).

A 'dispensação' é o ato de entrega do medicamento ao usuário na dose prescrita, na quantidade adequada e com informações suficientes para seu uso correto (BRASIL, 2009A). A sua execução começa no momento em que o usuário chega à farmácia, incluindo o momento de interação com o trabalhador, seja o próprio farmacêutico ou outro profissional. Esta fase de acolhimento é influenciada por fatores relacionados à qualidade da área de espera e, principalmente, pelas atitudes e pelos comportamentos do provedor quando se aproximam os usuários (ESHER ET AL., 2011). Por ser um momento de contato estreito com o usuário, esse componente foi relacionado à gestão do cuidado por se acreditar que as atividades realizadas nessa etapa devem ser desenvolvidas visando ao cuidado com o usuário.

Alguns componentes da gestão do cuidado alimentados pela dispensação são: educação em saúde; aconselhamento e medidas de prevenção; tomada de decisões clínicas, diagnósticas e terapêuticas; e a produção da informação. Os demais - como coordenação do cuidado, visão contínua dos processos, trabalho em equipe e relações 
interprofissionais, educação permanente e formação e orientação ao cidadão - podem ser desenvolvidos de forma independente ou em paralelo à dispensação.

O componente 'educação em saúde' reúne atividades educativas, de prevenção de riscos ligados ao uso de medicamentos, principalmente com doentes crônicos e crianças, utilizando ferramentas de trabalho com grupos, visita domiciliar e intervenção individual. Neste componente são propostas ações para a prevenção de riscos ligados à intoxicação infantil e ao descarte inadequado de medicamentos. Segundo Werneck (2009), as intoxicações exógenas são importantes causas de morbidade infantil no cenário mundial, representando uma carga alta tanto em termos de morbidade como no que diz respeito aos custos de atenção hospitalar. Isso leva a crer que ações de prevenção e promoção voltadas ao público infantil são uma importante estratégia a ser desenvolvida pelos Sefar.

O terceiro componente técnico, intitulado 'tomada de decisões clínicas, diagnósticas e/ou terapêuticas', possui um conjunto de ações voltadas para a clínica e a terapêutica do paciente. Diz respeito à identificação de erros de prescrição, elaboração de plano terapêutico compartilhado, identificação de problemas relacionados a medicamentos e consulta farmacêutica, caso necessário. Este componente sugere ao profissional farmacêutico um maior envolvimento com as questões diretamente ligadas à condição de saúde do usuário, bem como aos efeitos do tratamento sobre sua saúde individual.

Alguns estudos reforçam a ideia de que a intervenção do farmacêutico aumenta a adesão a regimes terapêuticos e pode promover redução de custos hospitalares, ao reduzir o número de prescrições, de internações e de medicamentos associados a reações adversas (ROMANO-LIEBER ET AL., 2002). Segundo resultado de estudo desenvolvido no Canadá por Mckinnon e Jorgenson (2009), houve uma melhora na gestão da medicação quando o farmacêutico estava integrado ao centro primário de saúde e colaborava com médicos de família na realização ou renovação da prescrição. Portanto, a presença do farmacêutico na tomada de decisões terapêuticas na APS é de suma importância para aprimorar o processo de cuidado e evitar danos à saúde, sendo que, para haver de fato reconhecimento desse trabalho, é preciso divulgar tais ações.

Para isso, a 'produção da informação' é considerada um componente extremamente importante do ponto de vista da gestão. A realização de ações farmacêuticas promotoras, principalmente, de resultados positivos em saúde deve ser alvo de estudos, a fim de gerar informações para o serviço e para a população. A participação em eventos técnicos precisa ser incentivada pelos gestores como forma de estimular a criatividade dos profissionais e o aprimoramento do processo de trabalho. Outras formas de comunicação também podem ser usadas no desenvolvimento de campanhas de comunicação social, com propósito de promover a discussão sobre medicamentos e seu uso racional. Além disso, materiais informativos auxiliam os usuários no autocuidado orientado e na automedicação responsável.

O mecanismo de comunicação é algo muito utilizado nos Sefar e contam com o desenvolvimento de habilidades específicas. O treinamento contínuo dos recursos humanos, principalmente dos profissionais atuantes nos Sefar, é algo que precisa estar inserido na rotina do profissional farmacêutico. A função 'educação permanente e formação' está prevista na Política de Atenção Básica como atribuição dos profissionais atuantes na atenção primária. Logo, não poderia deixar de estar presente no ML dos Sefar, uma vez que suas atividades, principalmente aquelas ligadas à formação profissional e autoavaliação, colaboram para a melhoria dos processos de trabalho em saúde.

O ML dos Sefar conta com componentes relacionados a diretrizes preconizados pela Política Nacional de Atenção Básica 
(PNAB) (2011B). A corresponsabilização pela coordenação do cuidado, a visão integral dos processos e assistências e o 'trabalho em equipe' são atitudes incorporadas ao ML dos Sefar por meio de atividades bem definidas, tendo como foco a melhoria dos resultados dos serviços prestados aos usuários. A 'coordenação do cuidado' pressupõe o acompanhamento do usuário pelos diversos pontos da RAS, e esse conjunto de ações também deve envolver a garantia de acesso a medicamentos especializados. Tais medicamentos são fornecidos pelo ente estadual e normalmente atendem a uma demanda de tratamentos dos níveis secundário e terciário da RAS.

Além dessas atividades, o componente de coordenação do cuidado também pressupõe a realização de acolhimento à demanda espontânea, ou seja, realizar escuta qualificada à situação/queixa dos usuários, inclusive daqueles não pertencentes ao território adscrito pela referida unidade, avaliando a melhor forma de orientá-los (GOMES ET AL., 2010). O acolhimento é uma ação que perpassa diversas atividades dos Sefar, sendo uma prática presente em todas as relações de cuidado, nos encontros reais entre trabalhadores de saúde e usuários, nos atos de receber e escutar as pessoas, podendo acontecer de formas variadas (BRASIL, 2011C). A recepção da farmácia na unidade de saúde é um local de livre acesso para a população e onde não devem haver barreiras impeditivas. Sendo assim, o Sefar torna-se um dos locais da unidade de saúde onde o acesso a um profissional de saúde é bastante facilitado, permitindo-lhe ser visto pelos usuários como uma das principais portas de acesso e informação aos serviços dentro da unidade de saúde.

Para a consolidação do Sefar como um locus de referência do cuidado para usuários e trabalhadores da saúde, o profissional farmacêutico e sua equipe necessitam ter uma 'visão contínua e integral dos processos', conhecendo a rede de atenção à saúde municipal, as linhas de cuidado e o processo de trabalho das equipes. A participação no desenho, na implantação e na melhoria dos processos e assistências, no âmbito local, legitima o farmacêutico como membro da equipe e envolve outros profissionais nas atividades ligadas à promoção do uso racional e ao acesso a medicamentos.

O último componente técnico da gestão do cuidado e do ML dos Sefar é a 'orientação ao cidadão'. Embora tenha sido considerado um componente técnico com uma atividade bem definida, preconizando a participação do farmacêutico nos espaços de controle social, o conceito atrelado a esse componente deve perpassar todo o ML. Afinal, espera-se que os profissionais compartilhem com o cidadão a tomada de decisões ao oferecerem informação oportuna, permitindo que ele participe e decida, com conhecimento, sobre seu processo assistencial. Faz também com que suas ações deem uma resposta individualizada às necessidades e expectativas de cada usuário, considerando sua opinião e seus direitos. Deve garantir a confidencialidade da informação à qual tem acesso e manter respeito à intimidade e à privacidade do usuário durante sua assistência. E, ainda, favorecer acessibilidade dos cidadãos e contribuir para resolver a demanda assistencial no tempo adequado (SEFAP, 2001).

A identificação dos interessados cumpriu o papel de esclarecer quais são as pessoas que precisam estar articuladas para que aconteça, de fato, a implantação mínima dos Sefar. Além dos atores da macro e da mesogestão, os profissionais da microgestão, como gerentes de unidades básicas, desenvolvem um papel decisivo no desempenho desses serviços. Sem o apoio dos gerentes, a realização das diversas atividades propostas não é priorizada, e estas acabam sendo esquecidas em meio à rotina das atividades logísticas. A participação comunitária é também fundamental para a consolidação dos Sefar na APS, pois é por meio das 
demandas geradas e pelo o reconhecimento dos resultados positivos que os gestores passam a apoiar e priorizar as ações ligadas ao uso de medicamentos.

Embora sejam reconhecidos como precípuos a participação ativa desses atores e o seu apoio nas demandas estruturais da implantação, é necessário frisar que a corresponsabilização e o compromisso ético do farmacêutico são fundamentais para a implantação dos Sefar nos municípios. O profissional farmacêutico é peça chave para fazer a intervenção acontecer, bem como para estreitar o elo entre usuários e as equipes de saúde. Sabe-se que organizar o cotidiano do trabalho nos Sefar é uma tarefa árdua, que envolve saberes diversos, como habilidades de comunicação, técnicas e psicológicas. Dessa forma, é preciso investir em formação e qualificação técnica e cultural com o intuito de aprimorar competências e treiná-las para o exercício na APS.

A construção do ML pode ser considerada como uma grande contribuição para a gestão, pois ele define o que deve ser realizado e qual a estrutura necessária para que os objetivos da intervenção sejam alcançados. O modelo proposto sugere desafios para a atuação do Sefar. Tal ferramenta poderá ser utilizada como instrumento indutor de práticas, não devendo ser um documento isolado, mas, sim, algo útil, capaz de auxiliar os farmacêuticos a conhecer suas tarefas e compreender o que se espera da intervenção. Assim, espera-se que possa contribuir como proposta norteadora de melhores práticas em serviço, atendendo a uma necessidade das organizações no cotidiano do trabalho na APS.

Enfim, a atuação na APS implica a relação com a equipe multiprofissional, o envolvimento e o conhecimento sobre o território e a compreensão do papel desenvolvido por cada profissional dentro das unidades de saúde. Atuar nesse cotidiano pode ser enfrentado como um desafio para os farmacêuticos, pois, durante anos, a função desse profissional esteve restrita à gestão técnica do medicamento.

Das sete etapas da avaliabilidade propostas por Trurston e Ramaliu (2005): 1. Delimitar o programa pela identificação de metas, objetivos e atividades que o compõem; 2 . Analisar os documentos; 3. Modelar a entrada de recursos, as atividades realizadas e os efeitos esperados, identificando os elos causais; 4. Identificar os interessados; 5. Explorar o programa, buscando compreender como ele funciona; 6. Desenvolver um modelo de programa avaliável; e 7. Obter os acordos necessários para prosseguir com a avaliação, consideramos ter cumprido integralmente as quatro primeiras e parcialmente a quinta e a sexta. A sétima não foi realizada.

Disso, resultam algumas limitações. $\mathrm{O}$ estudo, ainda que envolvesse uma gestora dos Sefar do município em questão, tendo como motivação o interesse da própria autora pelo tema, assim como a solicitação de vários dos interessados aqui incluídos, foi desenvolvido em ambiente acadêmico, com os desafios trazidos pelo ajuste da temporalidade deste com a da vida real. Buscou-se ser abrangente na seleção dos entrevistados, mas houve foco na macrogestão nos três níveis do sistema brasileiro, não tendo sido incluídos representantes de todos os interessados, sobretudo os da microgestão e os usuários que foram, portanto, identificados por terceiros. Em todos os casos, a fala dos atores será sempre situacional e histórica. De forma semelhante, a compreensão do programa, traduzida no ML apresentado, é sempre uma representação dada por um conjunto de atores, portanto, permeada por seus valores e ideologias. Não se avançou, durante o estudo aqui relatado, para a etapa mais operacional, dos acordos concretos para a implementação do monitoramento, incluindo a programação de recursos e a definição de responsabilidades, etapas em processo no momento de fechamento do texto. 


\section{Considerações finais}

A proposta de realizar um estudo de avaliabilidade dos Sefar na APS do município do Rio de Janeiro teve como principal finalidade contribuir para o desenvolvimento de uma ferramenta útil, capaz de subsidiar os gestores da $\mathrm{AF}$ e a atenção primária na tomada de decisões gerenciais em tempo oportuno, apontando evidências para redirecionamento e melhoria do Sefar na ESF.

Foram identificados os objetivos e as metas da intervenção, cujas definições locais se mostraram coerentes com a literatura seminal internacional quanto ao primeiro aspecto e incipientes e pouco claras no segundo. Este certamente é um aspecto a ser melhorado para guiar o monitoramento de forma adequada. Foram mapeados os atores e seu interesse na intervenção, aspecto a ser confirmado com a implementação do monitoramento. O ML oferece a guia dos recursos, atividades, efeitos e sua relação causal.

É importante destacar que os EA se justificam como etapa preparatória para uma avaliação (ou, no caso, monitoramento), inclusive apoiando a decisão quanto à sua pertinência, seu foco e sua tempestividade. O presente trabalho pretendeu relatar apenas a fase do EA, realizada com intenção preparatória para o monitoramento da intervenção em foco, o qual encontrava-se ainda em preparação até o fechamento do texto. Tendo em vista a grande relevância dos EA na literatura internacional sobre avaliação e sua ainda baixa abordagem na literatura internacional, acreditamos que nosso relato possa trazer contribuições para os ajustes dessa estratégia no contexto nacional.

\section{Referências}

ARAUJO, A. L. A.; UETA, J. M.; FREITAS, O. Assistência farmacêutica como um modelo tecnológico em atenção primária à saúde. Revista Ciências Farmacêuticas Básica e Aplicada, Araraquara, v. 26, n. 2, p. $87-92,2005$

BARDIN, L. Análise de Conteúdo. São Paulo: Edições $70,2011$.

BEZERRA, L. C. A; CAZARIN, G.; ALVES, C. K. A. Modelagem de Programas: da teoria à operacionalização. In: SAMICO, I. et al. Avaliação em Saúde: Bases Conceituais e Operacionais (Org.). Rio de Janeiro: Medbook, 2010.

BRASIL. Ministério da Saúde. Secretaria de Ciência, Tecnologia e Insumos Estratégicos, Departamento de Assistência Farmacêutica e Insumos Estratégicos. Assistência farmacêutica na atenção básica: instruções técnicas para sua organização. 2 ed. Brasília, DF: Ministério da Saúde, 2006. 100 p. (Série A. Normas e Manuais Técnicos)
Ministério da Saúde. Secretaria de Ciência, Tecnologia e Insumos Estratégicos, Departamento de Assistência Farmacêutica e Insumos Estratégicos. Diretrizes para estruturação de farmácias no âmbito do Sistema Único de Saúde. Brasília, DF: Ministério da Saúde, 2009a. 44 p. (Série A. Normas e Manuais Técnicos).

Ministério da Saúde. Secretaria de Atenção a Saúde, Departamento de Atenção Básica. Diretrizes do NASF (Núcleo de Apoio à Saúde da Família). Brasília, DF: Ministério da Saúde, 2009b. 160 p. (Série B. Textos Básicos de Saúde) (Cadernos de Atenção Básica; n. 27).

. Conselho Nacional de Secretários de Saúde (CONASS). Assistência Farmacêutica no SUS. Brasília, DF: CONASS, 2011a.

. Ministério da Saúde. Portaria MS/GM n ${ }^{\circ} 2488$, de 21 de outubro de 2011. Aprova a Política Nacional de Atenção Básica, estabelecendo a revisão de diretrizes e normas para organização da atenção básica, para a 
Estratégia de Saúde da Família (ESF) e o Programa de Agentes Comunitários de Saúde. Diário Oficial [da] União. Brasília, DF: Secretaria de Atenção à Saúde, Departamento de Atenção Básica, 2011b.

Ministério da Saúde. Secretaria de Atenção a Saúde. Departamento de Atenção Básica. Acolhimento à demanda espontânea. Brasília, DF: Ministério da Saúde, 2011c. p. 56 (Série A. Normas e Manuais Técnicos) (Cadernos de Atenção Básica, n. 28, v. 1).

CORRER, C. J.; OTUKI, M. F.; SOLER, O. Assistência farmacêutica integrada ao processo de cuidado em saúde: gestão clínica do medicamento. Revista PanAmazônica de Saúde, Ananindeua, v. 2, n. 3, p. 41-49, 2011.

CRUZ, M. M. Avaliação de Políticas e Programas de Saúde: contribuições para o debate. In: MATTOS, R. A.; BAPTISTA,T. W. F. (Org.) Caminhos para Análise das Políticas de Saúde. Rio de Janeiro: IMS, 2011.

ESHER, A. et al. Logic Models from an Evaluability Assessment of Pharmaceutical Services for People Living with HIV/AIDS. Ciência \&t Saúde Coletiva, Rio de Janeiro, v. 16, n. 12, p. 4833-4844, 2011.

GOMES, C. A. P. et al. A Assistência Farmacêutica na Atenção à Saúde. Belo Horizonte: FUNED, 2010.

MARIN, N. et al. (Org.). Assistência Farmacêutica para gerentes municipais. Rio de Janeiro: OPAS/OMS, 2003.

MARTINS, P. C. et al. Conselhos de Saúde e a Participação Social no Brasil: Matizes da Utopia. Physis: Revista de Saúde Coletiva, Rio de Janeiro, v. 18, n. 1, p.105-121, 2008.

MCKINNON, A.; JORGENSON, D. Pharmacist and physician collaborative prescribing: For medication renewals within a primary health center. Canadian Family Physician, Torornto, v. 55, n. 12, p. 85-91, 2009.

MENDES, E. V. O cuidado das condições crônicas na atenção primária à saúde: o imperativo da consolidação da estratégia da saúde da família. Brasília, DF: Organização Pan-Americana da Saúde, 2012.
MENDES, L. V. et al. Disponibilidade de medicamentos nas unidades básicas de saúde e fatores relacionados: uma abordagem transversal. Saúde em Debate, Rio de Janeiro, v. 38, n. especial, p. 109-123, 2014.

NATAL, S. et al. Estudo de Avaliabilidade da Rede de Formação de Recursos Humanos da Secretaria de Vigilância em Saúde do Ministério da Saúde. Cadernos de Saúde Coletiva, Rio de Janeiro, v. 18, n. 4, p. 560-571, 2010.

ORGANIZACION PANAMERICANA DE LA SALUD (OPS). Taller: "Servicios farmacéuticos basados en la atención primaria de salud". Santo Domingo: OPS, 2009.

Servicios farmacéuticos basados en la atención primaria de salud. Documento de posición de la OPS/ OMS. Washington, DC: OPS, 2013. (La Renovación de la Atención Primaria de Salud en las Américas, n. 6).

PESSÔA, L. R.; SANTOS, E. H. A.; TORRES, K. R. B. O. (Org.). Manual do Gerente: desafios da média gerência na saúde. Rio de Janeiro: ENSP, 2011.

RIO DE JANEIRO (Cidade). Secretaria Municipal de Saúde e Defesa Civil. Plano Municipal de Saúde do Rio de Janeiro, Rio de Janeiro: Secretaria Municipal de Saúde e Defesa Civil, 2009.

Secretaria Municipal de Saúde e Defesa Civil, Assessoria de Assistência Farmacêutica. Manual de Instruções, Diretrizes e Procedimentos Operacionais da Assistência Farmacêutica. Rio de Janeiro: Secretaria Municipal de Saúde e Defesa Civil, 2011.

RICIERI, M. C. et al. O farmacêutico no contexto da estratégia em saúde da família, que realidade é esta? Visão Acadêmica, Curitiba, v. 7, n. 2. 2006.

ROMANO-LIEBER, N. S. et al. Revisão dos estudos de intervenção do farmacêutico no uso de medicamentos por pacientes idosos. Cadernos de Saúde Pública, Rio de Janeiro v. 18, n. 6, p. 1499-1507, 2002.

SOCIEDADE ESPAÑOLA DE FARMACÉUTICOS DE ATENCIÓN PRIMARIA (SEFAP). Competencias para 
farmacéuticos que trabajan en atención primaria. In: Series Divulgativas. Barcelona: SEFAP, 2001.

THURSTON, W. E.; RAMALIU, A. Evaluability assessment of a survivors of torture program: lessons learned. The Canadian Journal of Program Evaluation, Renfrew, v. 20 , n. 2, p. 1-25, 2005 .

VIEIRA, F. S. Possibilidades de contribuição do farmacêutico para a promoção da saúde. Ciência \&્t Saúde Coletiva, Rio de Janeiro, v. 12, n. 1, p. 213-220, 2007.

WERNECK, G. L.; HASSELMANN, M. H. Intoxicações exógenas em crianças menores de seis anos atendidas em hospitais da região metropolitana do Rio de Janeiro. Revista Associação Médica Brasileira, São Paulo, v. 55, n. 3, p. 302-307, 2009.
ZULUAGA, G. C. R. A assistência farmacêutica e a atenção primária à saúde: coordenação, integralidade e continuidade do cuidado na Dispensação e Atenção Farmacêutica no Brasil. 2013. Dissertação (Mestrado em Saúde Pública) - Fundação Oswaldo Cruz, Escola Nacional de Saúde Pública Sérgio Arouca, Rio de Janeiro, 2013.

\footnotetext{
Recebido para publicação em julho de 2014

Versão final em janeiro de 2015

Conflito de interesse: inexistente

Suporte financeiro: não houve
} 